\title{
François Cheng à la croisée de la Chine et de l'Occident, éd. Madeleine Bertaud et Cheng Pei
}

Laura Colombo

\section{(2) OpenEdition}

\section{Journals}

\section{Edizione digitale}

URL: http://journals.openedition.org/studifrancesi/5436

DOI: $10.4000 /$ studifrancesi.5436

ISSN: 2421-5856

\section{Editore}

Rosenberg \& Sellier

\section{Edizione cartacea}

Data di pubblicazione: 1 dicembre 2016

Paginazione: $565-566$

ISSN: 0039-2944

\section{Notizia bibliografica digitale}

Laura Colombo, «François Cheng à la croisée de la Chine et de l'Occident, éd. Madeleine Bertaud et Cheng Pei », Studi Francesi [Online], 180 (LX | III) | 2016, online dal 01 janvier 2017, consultato il 18 septembre 2020. URL : http://journals.openedition.org/studifrancesi/5436 ; DOI : https://doi.org/ $10.4000 /$ studifrancesi.5436

Questo documento è stato generato automaticamente il 18 settembre 2020.

\section{(c) (i) $\odot$}

Studi Francesi è distribuita con Licenza Creative Commons Attribuzione - Non commerciale - Non opere derivate 4.0 Internazionale. 


\title{
François Cheng à la croisée de la Chine et de l'Occident, éd. Madeleine Bertaud et Cheng Pei
}

\author{
Laura Colombo
}

\section{NOTIZIA}

François Cheng à la croisée de la Chine et de l'Occident, Madeleine BERTAUD et Cheng PEI (éd.), Genève, Droz, 2014, 255 pp.

1 «La beauté est une rencontre», ricorda giustamente François CHENG, e i Miroirs croisés tra Francia e Cina, tra mostre, convegni e pubblicazioni, hanno conosciuto un notevole sviluppo e rinnovamento negli ultimi anni, anche a livello di critica letteraria.

2 A venire qui approfondito è un caso unico nella letteratura francese, quello di Cheng Chi-sieng, diventato François nel battesimo, arrivato dalla Cina nell'Esagono all'età di diciannove anni, entrato, «comme irrésistiblement, dans la langue française», e non solo passeur ma créateur di cultura, cesellatore di una lingua volontariamente assunta, «dans un mariage d'amour et de raison» (p. 50), e di cui diventa infine legislatore, con l'elezione all'Académie française nel 2001. Autore di romanzi, saggi e soprattutto di raffinate raccolte poetiche, François Cheng è l'oggetto di questo volume che ospita una selezione delle comunicazioni presentate in due giornate di studi svoltesi presso la Bibliothèque nationale di Parigi e l'Università Fudan di Shanghai.

Dopo l'Ouverture di Bruno RACINE, presidente della BnF, l'Introduction pone gli enjeux della pubblicazione, che riunisce ricercatori della «terre natale» e della «terre d'accueil» dello scrittore al fine di «offrir pour la première fois une lecture croisée de l'œuvre» (p. 11), non cronologica ma sulla base di risonanze tematiche.

Quattro sono le parti che compongono il volume. La prima, «Quand je devient un autre», comprende un contributo di ZHANG Hua che, occupandosi della «quête identitaire» attraverso Le Dit de Tiany, analizza la polifonia dell'enunciazione che rinvia al legame 
tra l'io e l'altro, tra l'autore e il suo personaggio, sotto il segno del "tre" taoista, yin, yang e «vide médian». CHU Xiaoquang riprende e precisa questa «immense potentialité du trois» (p.44) nello stesso «langage romanesque» che, come in certi quadri cinesi, lascia al lettore e alla sua immaginazione il compito di «combler les vides» (p.47), mentre la maestosa confluenza di figure e significati non saprebbe non rinviare al roman-fleuve Jean-Christophe di Romain Rolland (p. 48), peraltro tra i primi testi tradotti in Cina dal grande Fu Lei. oIN Haying, in François Cheng entre le propre et l'étranger, allude alla famosa formulazione di Antoine Berman per sottolineare l'«apprentissage du signe» (p. 53) fatto da Cheng, venuto da un mondo di segni ideogrammatici e vicino per un periodo allo strutturalismo francese, per far proprio quello che gli era prima étranger, rinviando in particolare al Dialogue.

Una seconda sezione, intitolata «Habiter poétiquement la terre», riguarda la poesia di Cheng, parte preponderante della sua produzione. Più di mezzo secolo dopo l'Orphée noir sartriano, Cheng PEI si mette «à l'écoute de l'Orphée sorti d'ailleurs», continuando l'assimilazione del poeta straniero alla figura antonomastica della poesia europea, e sottolineando l'importanza dell'intertesto cinese nel virtuosismo di immagini e risonanze dei suoi "ideogrammi" occidentali. Dominique MILLET-GIRARD prosegue questa vena indagando le affinità delle forme brevi di cheng e Claudel, nel loro «haut orphisme croisé et partagé» (p. 85), mentre Pierre BRUNEL attraversa Vraie lumière née de vraie nuit convocando tanto la Genesi e Péguy quanto Rimbaud, Claudel e Rilke, Baudelaire e Bonnefoy o Richard Strauss, in una raffinata analisi ricca di temi e spunti cosmogonici. Luc FRAISSE mostra come le categorie critiche occidentali siano insufficienti ad analizzare la poetica del romanzo di Cheng, quasi epifenomeno della sua poesia che richiede «une lecture intuitive, une lecture d'acclimatation» (p. 105), conducendo a una molteplicità di interpretazioni per esaurire le quali «l'éternité n'est pas de trop» (p.115). Infine, Madeleine BERTAUD si interessa del féminin, eterna questione riportata più tradizionalmente in François Cheng a «une intimité avec l'univers», privilegiata peraltro dal taoismo, di questi «êtres de rythme» (p.121), quando il «mystère spécifique à la femme» (p.119) tra «maternité et compassion» (p.125), non la priva «de sa réalité charnelle ni de sa présence spirituelle», ma la rende «profondément complémentaire» all'uomo (p. 131).

6 La terza parte, «Eil ouvert et cœur battant», è dedicata ai rapporti di Cheng con la pittura, cinese e occidentale. Lise SABOURIN sottolinea come, nella sua «quête de beauté», il suo testo sia «toujours pictural» (p.136) e la sua poetica «synesthésique par essence» (p. 146). Éric LEFEBVRE si occupa degli scritti sulla pittura cinese di Cheng, ricordando quanto l'analisi del "sostrato" pittorico sia un fatto occidentale, Cheng avendo conosciuto le opere della pittura cinese soprattutto dopo il suo arrivo in Francia. In un secondo contributo, lo stesso LEFEBVRE si sofferma in particolare su Shitao. In «Poésie et peinture: Cheng et Claudel», Dominique MILLET-GÉRARD rende omaggio alla loro «intelligence comparative», quando Cheng giunge a esplicitare le intuizioni claudeliane, nella «commune et passionnée quête du sens» (p. 181), mentre Madeleine BERTAUD ritorna su «François Cheng à la rencontre de la peinture occidentale, retrouvaille et découvertes (Vinci, Cézanne, Rembrandt)», rinviando tra gli altri al Pèlerinage au Louvre.

7 L'ultima sezione, «D'un signe à l'autre», costituisce un'interessante e originale disanima dei problemi traduttivi posti da questo originale dia-logismo franco-cinese, operante nei due sensi. In una «Note sur la traduction chinoise du Dit de Tianyi», Cheng PEI, 
utilizzando ancora le formulazioni bermaniane, analizza la singolarità del concetto di scarto, reperibile anche nella lingua "originale", ma non nativa, del romanzo. YUAN Li prosegue queste riflessioni nella direzione complementare delle traduzioni di poesie cinesi da parte di Cheng, estendendo una delle parole chiavi di Berman, l'etica, dalla traduzione a «une éthique planétaire» (p.212) e convocando anche alcune riflessioni bakhtiniane sull'evoluzione del senso che si rinnova "avec l'évolution du dialogue» (p. 213). zHU Jing, in «Un dialogue du cœur au cœur (à propos de ma traduction d'À l'Orient de tout)», riporta il suo incontro con il calligrafo, quando «le souffle devient signe» e il segno, riprodotto in queste pagine, è reinterpretato dalle parole. Una microstoria della traduttologia cinese si affianca a questo dialogo interculturale e intersemiotico, in cui un poeta cinese è intervenuto a "perfezionare" la trasposizione dell'alfabeto in ideogramma.

8 La miscellanea si completa con una Postface a due voci dei curatori, che non è tra i contributi critici meno preziosi per la comprensione generale della figura e dell'opera di François Cheng.

9 Certamente destinato agli specialisti, ma adatto anche a una densa introduzione all'opera di François Cheng, il volume ha il merito di presentare letture puntuali e approfondite dei suoi diversi aspetti con un occhio particolare rivolto alla Cina, allo stesso tempo punto di partenza e di arrivo, come dimostra anche la massiccia presenza di trascrizioni in mandarino, in nota e nel paratesto. Ma proprio per questo, si tratta di un interessante viaggio che lega in modo relativistico radici ed esotismo, facendo di entrambi gli "imperi culturali" dei «vrai[s] pays de gloire», come avrebbe detto Baudelaire. 